\title{
Transcranial light-emitting diode therapy for neuropsychological improvement after traumatic brain injury: a new perspective for diffuse axonal lesion management
}

This article was published in the following Dove Press journal: Medical Devices: Evidence and Research

\author{
João Gustavo Rocha \\ Peixoto dos Santos \\ Wellingson Silva Paiva \\ Manoel Jacobsen Teixeira
}

Department of Neurological Surgery, University of São Paulo School of

Medicine, São Paulo, Brazil
Correspondence: João Gustavo Rocha Peixoto dos Santos

Department of Neurological Surgery, University of São Paulo School of

Medicine, 255 Dr. Enéas de Carvalho Aguiar Avenue, 05403-010 São Paulo, Brazil

$\mathrm{Tel}+55$ II 941989876

Email joao.gustavo.rps@gmail.com

\begin{abstract}
The cost of traumatic brain injury (TBI) for public health policies is undeniable today. Even patients who suffer from mild TBI may persist with cognitive symptoms weeks after the accident. Most of them show no lesion in computed tomography or conventional magnetic resonance imaging, but microstructural white matter abnormalities (diffuse axonal lesion) can be found in diffusion tensor imaging. Different brain networks work together to form an important part of the cognition process, and they can be affected by TBI. The default mode network (DMN) plays an important central role in normal brain activities, presenting greater relative deactivation during more cognitively demanding tasks. After deactivation, it allows a distinct network to activate. This network (the central executive network) acts mainly during tasks involving executive functions. The salience network is another network necessary for normal executive function, and its activation leads to deactivation of the DMN. The use of red or near-infrared (NIR) light to stimulate or regenerate tissue is known as photobiomodulation. It was discovered that NIR (wavelength 800-900 nm) and red (wavelength $600 \mathrm{~nm}$ ) light-emitting diodes (LEDs) are able to penetrate through scalp and skull and have the potential to improve the subnormal, cellular activity of compromised brain tissue. Based on this, different experimental and clinical studies were done to test LED therapy for TBI, and promising results were found. It leads us to consider developing different approaches to maximize the positive effects of this therapy and improve the quality of life of TBI patients.
\end{abstract}

Keywords: traumatic brain injuries, diffuse axonal injury, low-level light therapy, neurologic manifestations, post-concussion syndrome, quality of life

\section{Current impact of traumatic brain injury (TBI) for public health policies}

In the US, there occur 50,000 deaths annually from TBI, with the majority of those on the scene. Of an annual emergency room attendance of 1.1 million, there are 235,000 hospitalizations from nonfatal TBI. Recently, there has been an increase in TBIs presenting to the emergency department by $14 \%{ }^{1,2}$

Despite most of the victims of TBI recovering satisfactorily, some studies estimated higher rates (some with the considerable rates of $70 \%-90 \%$ ) of these patients presenting with psychological or neurological symptoms weeks or months after the original head trauma ${ }^{3}$ and $5 \%-22 \%$ of them persisting with these cognitive problems after these initial weeks. ${ }^{4}$ Most of these patients present with mild TBI associated with normal computed tomography (CT) findings and no post-traumatic amnesia, and they 
normally present with complete resolution of post-traumatic symptoms within 1 month. However, $\sim 30 \%$ of those with mild TBI and post-traumatic amnesia have persistent posttraumatic symptoms, and a significant number at 1-year postinjury have decreased functional outcome and disability. ${ }^{5}$ The current data indicate that $\sim 124,000$ (43.1\%) of people discharged with TBI from acute hospitalizations developed TBI-related long-term disability. ${ }^{6}$ The definition of disability was given broadly, including inability or considerable difficulty in performing the activities of daily living, presenting post-injury symptoms, which prevented people from doing things they intended to do, and poor cognitive and mental health levels on standard measures focused on the findings from a previous population-based study. ${ }^{7}$

Despite these relevant rates, it is important to emphasize that they are likely underestimated, once they depend on hospitalizations only, and they do not include TBI treated in other settings or for which treatment was not sought. ${ }^{6}$

\section{Relationship of TBI, neuropsychological disorders and radiological features}

Neuropsychological disorders include sleep problems (in 53\% of patients), ${ }^{4}$ irritability, apathy ${ }^{8}$ and even diseases such as depression, obsessive-compulsive disorder (OCD) and anxiety syndromes..$^{8-10}$ Other common symptoms include headaches, balance problems, dizziness, fatigue and memory and attention difficulties. ${ }^{10}$ This constellation of physical, emotional and cognitive symptoms is included in formal diagnostic criteria for postconcussion syndrome. ${ }^{10}$ These presentations affect every aspect of an individual's life, such as education, potential employment opportunities and even social and family relationships. ${ }^{11}$

Most brain lesions leading to these symptoms are not shown in structural CT or magnetic resonance imaging (MRI) ${ }^{4,12-14}$ However, abnormalities in the white matter (diffuse axonal lesion) could be found in diffusion tensor imaging (DTI) MRI scans. ${ }^{13,15}$ DTI is sensitive to detect water diffusion in the brain. Thus, DTI is used to find microstructural lesion in white matter tracts. ${ }^{16}$ There are five white matter regions of interest where one can usually see lesions:

1. anterior corona radiata ( $41 \%$ of TBI cases),

2. uncinate fasciculus ( $29 \%$ of TBI cases),

3. genu of corpus callosum $(21 \%)$,

4. inferior longitudinal fasciculus $(21 \%)$ and

5. cingulum bundle $(18 \%) .{ }^{17}$

Almost all the symptoms and signs following TBI are attributed to the loss of connectivity in the aforementioned tracts. ${ }^{12}$

\section{Physiopathology of neurophysiological disorders after TBI}

Increasing focus has been directed to brain regions in which neural activity is greater during the baseline state than during an experimental task. This state is called deactivation. The finding that demonstrates such task-related relative decreases in activity across a broad range of cognitive tasks when compared with a baseline state is what reinforces the current attention to this default mode theory. Thus, the default mode network (DMN) forms an important part of the cognition process. ${ }^{18}$ Disruption of the DMN is clinically important, and abnormalities are observed in various neurological and psychiatric disorders. ${ }^{19}$

The power to regulate DMN functions seems to be a central point of normal brain activity, presenting greater relative deactivation seen during more cognitively demanding tasks. ${ }^{20}$ Problems associated with sustained attention after TBI, as revealed by behavioral inconsistency, are related to an increase in DMN activity. ${ }^{21} \mathrm{DMN}$ as two other highly specialized brain networks is important for cognition. Frequently, they have been seen dysregulated on resting-state functional connectivity magnetic resonance imaging (fMRI) scans in TBI. ${ }^{4}$

The DMN can be divided as follows ${ }^{22}$ :

1. anterior areas in the medial frontal lobes (medial prefrontal cortex [mPFC]);

2. posterior areas in the medial parietal lobes (precuneus and posterior cingulate cortex [precun/PCC]) and posterolateral areas in the lateral parietal lobes (angular gyri) and

3. deep, medial temporal lobes/hippocampal areas.

The medial posterior portion of DMN (precun/PCC) needs to deactivate for normal cognitive function to occur. Thus, a distinct network called the central executive network (CEN) is used that can activate. ${ }^{21}$

The CEN is another network involved in the cognition process and is basically composed of two areas ${ }^{4}$ :

1. the dorsolateral prefrontal cortex and

2. the posterior parietal cortex (mainly intraparietal sulcus area).

The CEN is a part of network that activates during functional MRI tasks involving executive functions (as salience network [SN]). Its activity is anticorrelated with DMN function. ${ }^{22}$

The last network affected in TBI cases is the SN. It consists of three areas ${ }^{4}$ : 
1. anterior insulae;

2. presupplementary motor areas and

3. dorsal anterior cingulate cortex.

The SN is necessary for normal executive function and inhibition. It controls the DMN. ${ }^{23,24}$

A couple of papers studied the effects of near-infrared (NIR) light to improve cognition through inducing a brain resting state. NIR light promoted an increase in power in alpha, beta and gamma frequencies in electroencephalogram (EEG). These findings are consistent with a brain resting stage, which is implicated in improved cognition..$^{25,26}$

\section{Principles of red/NIR transcranial light-emitting diode (tLED) photobiomodulation (PBM)}

The process of using red or NIR light to stimulate or regenerate tissue is known as PBM. It was accidentally found out after the Hungarian Endre Mester tried to use laser beams (trying to repeat an experiment performed in Boston, Massachusetts) to destroy cancer that was experimentally implanted into a laboratory rat. ${ }^{27}$ The ruby laser that had been built was only a tiny fraction of the power of the laser that had previously been used in Boston by McGuff, ${ }^{28}$ and he observed hair regrowth and wound healing in the stimulated area where tumor had been implanted. ${ }^{27}$

More than 30 years ago, it was also observed in cadaver studies that NIR (wavelength 800-900 nm) and red (wavelength $600 \mathrm{~nm}$ ) LEDs are able to penetrate through scalp and skull $(2 \%-3 \%, \sim 1 \mathrm{~cm})^{29,30}$ and have the potential to improve the subnormal, cellular activity of compromised brain tissue.

Currently, PBM is accepted as a therapeutic tool for infected, ischemic and hypoxic wounds and also presents positive effects in healing, reducing edema and inflammation, relieving pain, treating chronic inflammation and autoimmune diseases and recovering from ischemic heart disease and even attenuating the degeneration of optic nerve. ${ }^{31,32}$ There are multiple studies for other pathological processes as TBI in course. , $27,33,34^{-}$

Optimal therapeutic intervention was characterized by wavelengths within the far-red to NIR range (630-1064 nm) along with a minimal energy density of $4 \mathrm{~J} / \mathrm{cm}^{2} .{ }^{35}$ These wavelengths and energy density were proven to be effective at stimulating biological processes, and they act by four main actions:

1. Increasing adenosine triphosphate (ATP) production in the mitochondria. ${ }^{36,37}$ Which happens due to cytochrome c oxidase (CCO) increased effect. CCO absorbs light photon and avoids nitric oxide (NO) inhibitory effect on itself. Thus, the mitochondrial membrane potential is increased; there will be more oxygen consumed, as soon as more glucose is metabolized and more production of ATP happens. ${ }^{36}$

2. Increasing regional cerebral blood flow. ${ }^{37,38}$

3. An anti-inflammatory effect by inhibiting microglial activation and also strong antioxidant effects (increase in mitochondrial superoxide dismutase). ${ }^{39,40}$

4. Signaling mediators and activation of transcription factors that cause changes in protein expression that last for some considerable time and explain long-lasting effect. ${ }^{28}$

5. Increasing neurogenesis and synaptogenesis (described in small animal studies treated with NIR in the acute-stage post-TBI). ${ }^{34,40}$

Besides these biological effects, the role of heating during transcranial laser therapy to influence the cognitive improvement results had not been investigated. To address this potential confounding factor, a recently published paper $^{41}$ studied 11 patients in whom a heat generator produced the same timedependent temperature alteration curve which the transcranial laser system produced at the same forehead location. A monitor was used to evaluate hemodynamic and metabolic changes in the forehead tissue near the thermal stimulation site before, during and after the heat stimulation. The results showed that $\mathrm{CCO}$ of the forehead tissue was not modified for this heat stimulation, and oxyhemoglobin changes were observed in comparison with laser stimulation. The authors concluded that heat was not responsible for cerebral hemodynamics and metabolic changes during transcranial laser therapy. ${ }^{41}$

The differentiation of a progenitor cell into a neuron is called neurogenesis. It usually occurs in the subventricular zone and hippocampus. It is not such a rare event in the brain as we once thought it was. ${ }^{42}$ The formation of new synapses is the process called synaptogenesis. It is one of the brain recovery mechanisms after TBI. ${ }^{43}$ NIR transcranial photobiomodulation (tPBM) was used to treat mice with acute severe TBI in current small studies, and these studies have supported the notion that tPBM increases neurogenesis and synaptogenesis. Beginning the protocol $4 \mathrm{~h}$ post TBI, they found that there was a significantly better recovery at 28 days post TBI in mice receiving NIR tPBM treatments thrice daily when compared to controls. The neurogenesis was noted to be increased when measured by immunofluorescence staining of brain sections. ${ }^{44}$

The respiratory mitochondrial enzyme $\mathrm{CCO}$ is the primary infrared photoacceptor in cells. Its increased activity after PBM is followed by increased hemoglobin oxygenation, explaining its role on neuromodulation. ${ }^{45}$

The brain imaging technique established compared to functional MRI is functional NIR spectroscopy (fNIRS), 
which uses 700-900 nm light. ${ }^{46}$ The estimated mean of penetration depth (5\% remaining intensity) of NIR light through the scalp and skull is $23.6 \pm 0.7 \mathrm{~mm} .{ }^{47-49}$ Penetration also depends on the anatomical region of the skull and wavelength. ${ }^{50,51}$ Wavelength is the most important parameter that governs the depth of penetration of laser light into tissue. Living tissues' absorption and scattering coefficients are higher at lower wavelength; thus, NIR light penetrates more deeply that red light and so on. ${ }^{52}$ In tPBM application, NIR light is frequently used on the forehead (penetration is easier - no hair, longer wavelength). ${ }^{27}$

The clinical effects of PBM were tested in many studies. A controlled study with 20 patients (and $>20$ patients for the control group) using low-level laser stimulation (1024 nm wavelength) could demonstrate enhancement in sustained attention and short-term memory. ${ }^{53}$

With a similar approach, another paper was published using a low-level laser therapy with a specific wavelength (1024 nm) in 15 controlled patients. Its results suggested that transcranial laser stimulation improves executive function and may have exciting potential for treating neuropsychological disorders deficits. ${ }^{54}$

Two types of category learning structures have been studied: rule based and information integration. These category learning structures are related to two dissociable neural systems, and both rely on the network of brain regions: a reflective system (optimal for rule-based structures and highly reliant on processing on prefrontal cortex) and a reflexive system (optimal for information integration and reliant on processing on striatum). Testing the effects of transcranial infrared laser stimulation on these systems, it was found that prefrontal rule-based learning was improved as compared to placebo, but the same did not happen for striatum information-integration learning as compared to placebo. ${ }^{45}$

\section{Experimental and clinical studies with red/NIR tLED for TBI}

The first described TBI animal model treated with tLED therapy was done with mice, which were subjected to head cerebral contusion and received $808 \mathrm{~nm}$ NIR/red lightemitting diode (LED). Only one tLED section $4 \mathrm{~h}$ after the injury reduced a considerably size of the post-injury area by $90 \%$, and neurological severity scores were significantly lower at 28 days for laser-treated mice. ${ }^{55}$ Mice receiving $6 \mathrm{~min}$ daily tLED session for 10 days reverted deficits in working memory tests. ${ }^{56}$

An open protocol with PBM in a two-case study with tLED designed to improve cognitive function in chronic mild
TBI patients also found considerable improvements in verbal learning, executive function and memory. ${ }^{17}$

Increasing the number of patients with chronic mild TBI, the same author of the previous quoted study published positive results for 11 patients submitted to 18 outpatient sessions, starting at 10 months to 8 years post TBI. Each LED cluster head $\left(5.35 \mathrm{~cm}\right.$ diameter, $\left.500 \mathrm{~mW}, 22.2 \mathrm{~mW} / \mathrm{cm}^{2}\right)$ was applied during 10 minutes to every 11 scalp placements $\left(13 \mathrm{~J} / \mathrm{cm}^{2}\right)$. Improvements were evidenced in executive function and sleep and fewer post-traumatic stress disorders. ${ }^{57}$

Other series of two patients submitted to $18 \mathrm{red} / \mathrm{NIR}$ tLED treatments $\left(500 \mathrm{~mW}, 22.2 \mathrm{~mW} / \mathrm{cm}^{2}, 22.48 \mathrm{~cm}^{2}\right.$ per treatment area) in chronic TBI patients (who began tLED at 10 months- 8 years post TBI) showed improvements in cognition (mainly executive function and verbal memory). ${ }^{58}$

Resting state fMRI scans have been obtained before and after $18 \mathrm{tLED}$ treatments $\left(26 \mathrm{~J} / \mathrm{cm}^{2}\right.$ per LED cluster head placement red/NIR, $500 \mathrm{~mW}, 22.2 \mathrm{~mW} / \mathrm{cm}^{2}$ ) in left-hemisphere stroke patients with chronic aphasia, which showed significant increases in "naming ability" and significantly increased correlations between pairs of cortical nodes within each of three separate networks (DMN, CEN, SN) post tLED. ${ }^{4}$

Another study using a high-power NIR laser (10-15 W at 810 and $980 \mathrm{~nm}$ ) to treat a moderate TBI patient through 20 NIR applications over a 2-month period showed decreased headache, depression, anxiety and insomnia, whereas cognition and quality of life improved, accompanied by changes in the single-photon emission computed tomography (SPECT) imaging. ${ }^{59}$ A summary of these studies is given in Table 1.

It is important to emphasize that these clinical studies with LED therapy for TBI were neither controlled nor randomized. Thus, their conclusion may simply be due to chance. Randomized, controlled studies are necessary for further conclusions.

Safety of PBM was tested in different studies. One of the first concerns was the cortical temperature during stimulation. A rabbit study in which NIR laser therapy (continuous wave energy $15 \mathrm{~J} / \mathrm{cm}^{2}$ at $808 \mathrm{~nm}$ wavelength) was applied for $10 \mathrm{~min}$ showed a cortical temperature increase under the laser probe by $0.8^{\circ} \mathrm{C}-1.8^{\circ} \mathrm{C}$ during treatment. ${ }^{60}$ Human studies were also performed to test the safety of this therapy, and no adverse events could be related to PBM. ${ }^{61,62}$ These studies and others describe no short- or long-term detrimental effects. ${ }^{17,57,58}$ Low-level laser system (PBM) is registered in the Food and Drug Administration, and adverse effects described are ocular injuries, electrical shock and unintended cell damage. ${ }^{17,57,63}$ 


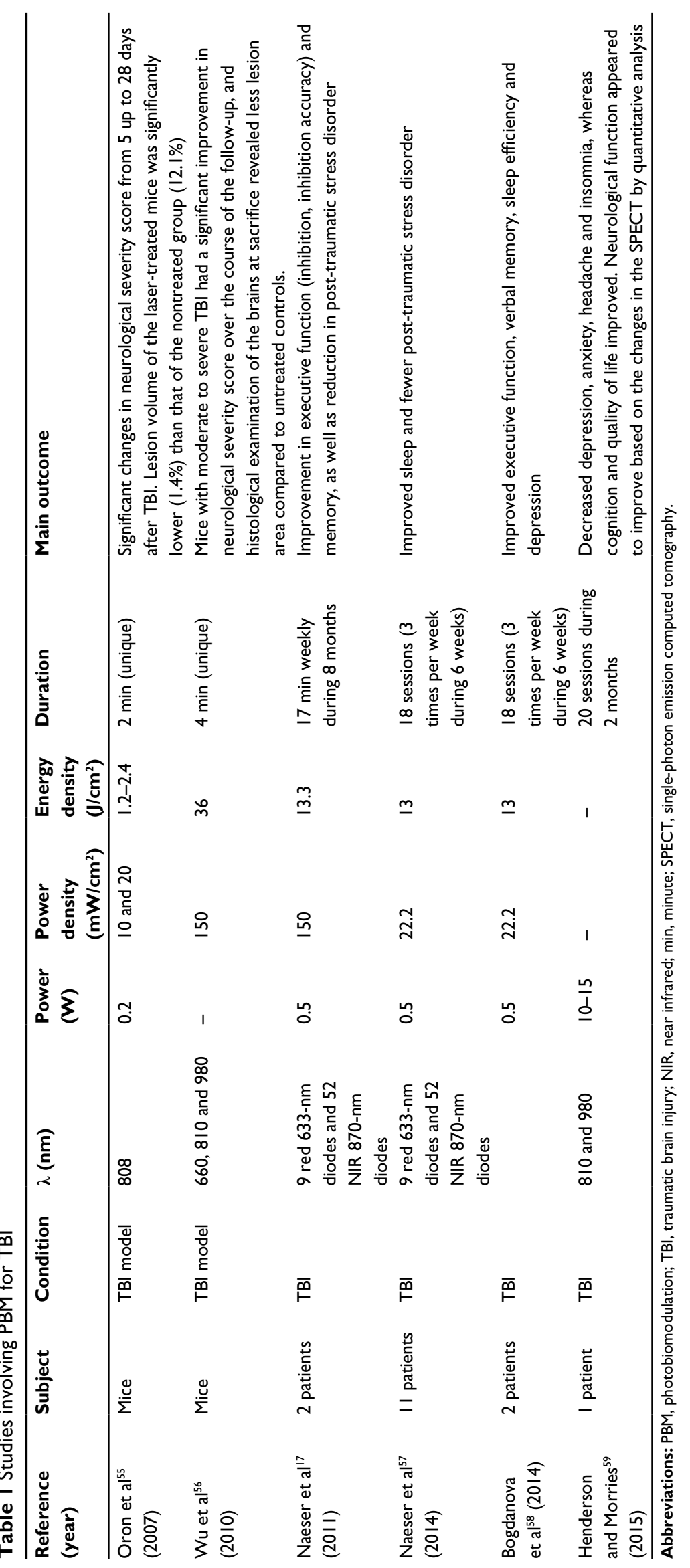




\section{Conclusion}

It is essential to have a rehabilitation plan, considering the morbidity due to TBI, preferably multi-professional, in order to get the maximum degree of recovery of neuropsychological activities. tLED therapy is an interesting approach to this, since it allows researchers to study the activity of the human brain in real time and finally to guide the patient's brain to plastic changes. ${ }^{64}$

There are different approaches for treatment of neuropsychological symptoms after TBI beyond PBM. Pharmacological treatments target the modulation of major neurotransmitter systems - dopaminergic, serotonergic, noradrenergic, acetylcholinergic and glutamatergic. Serotonin reuptake inhibitors act for depression secondary to TBI. Modulation of the dopaminergic system improves alertness, attention and cognitive processing speed. Cognitive and memory impairments may reflect the disruption of cholinergic function, and the effects of anticholinergic agents support this contention. ${ }^{65}$

Repetitive transcranial magnetic stimulation (rTMS) induces changes in cortical excitability, leading to reorganization of the network responsible for impaired function. ${ }^{64}$ Some studies have found cognitive improvements after rTMS. ${ }^{66}$ Transcranial direct current stimulation is another therapy for TBI rehabilitation based on brain excitability modulation via low-amplitude (0.5-2 mA) direct current through the scalp, and Demirtas-Tatlidede et al demonstrated its role in the facilitation of several cognitive domains. ${ }^{67}$

There is still a lot to understand and study about this new technology (PBM), but it is undeniable that its results are interesting so far. There are many questions that need to be elucidated such as duration of treatment, when to start and if all TBI patients should be treated or only a few of them depending on their characteristics. Answers like these may only be available after randomized clinical trials. However, the displayed data demonstrate that tLED therapy may be considered as a promising treatment for improvement in the quality of life of TBI patients.

\section{Acknowledgments}

This research did not receive any specific grant from funding agencies in the public, commercial or not-for-profit sectors.

\section{Disclosure}

The authors report no conflicts of interest in this work.

\section{References}

1. Langlois JA, Rutland-Brown W, Thomas KE, Xi YL. Incidence of traumatic brain injury in the United States, 2003. J Head Trauma Rehabil. 2006;21(6):544-548.
2. Centers for Disease Control and Prevention [webpage on Internet]. Atlanta: National Center for Injury Prevention and Control [updated January 22, 2016; cited January 22, 2016]. Available from: https://www. cdc.gov/traumaticbraininjury/data/rates.html. Accessed October 17, 2017.

3. Shafiei E, Fakharian E, Omidi A, Akbari H, Delpisheh A. Effect of mild traumatic brain injury and demographic factors on psychological outcome. Arch Trauma Res. 2016;5(2):e29729.

4. Naeser MA, Hamblin MR. Traumatic brain injury: a major medical problem that could be treated using transcranial, red/near-infrared LED photobiomodulation. Photomed Laser Surg. 2015;33(9):443-446.

5. Dikmen S, McLean A Jr, Temkin NR, Wylen AR. Neuropsychologic outcome at one-month postinjury. Arch Phys Med Rehabil. 1986;67(8):507-513.

6. Corrigan JD, Selassie AW, Orman JA. The epidemiology of traumatic brain injury. J Head Trauma Rehabil. 2010;25(2):72-80.

7. Selassie AW, Zaloshnja E, Langlois JA, Miller T, Jones P, Steiner C. Incidence of long-term disability following traumatic brain injury hospitalization in the United States. J Head Trauma Rehabil. 2008;23(2):123-131.

8. Alway Y, McKay A, Ponsford J, Schönberger M. Expressed emotion and its relationship to anxiety and depression after traumatic brain injury. Neuropsychol Rehabil. 2012;22(3):374-390.

9. Schönberger M, Ponsford J, Gould KR, Johnston L. The temporal relationship between depression, anxiety, and functional status after traumatic brain injury: a cross-lagged analysis. J Int Neuropsychol Soc. 2011;17(5):781-787.

10. Donnell AJ, Kim MS, Silva MA, Vanderploeg RD. Incidence of postconcussion symptoms in psychiatric diagnostic groups, mild traumatic brain injury, and comorbid conditions. Clin Neuropsychol. 2012;26(7):1092-1101.

11. Yeates KO, Taylor HG, Walz NC, Stancin T, Wade SL. The family environment as a moderator of psychosocial outcomes following traumatic brain injury in young children. Neuropsychology. 2010;24(3):345-356.

12. Niogi SN, Mukherjee P, Ghajar J, et al. Extent of microstructural white matter injury in postconcussive syndrome correlates with impaired cognitive reaction time: a $3 \mathrm{~T}$ diffusion tensor imaging study of mild traumatic brain injury. AJNR Am J Neuroradiol. 2008;29(5):967-973.

13. Lipton ML, Gellella E, Lo C, Gold T, Ardekani BA, Shifteh K, Bello JA, Branch CA. Multifocal white matter ultrastructural abnormalities in mild traumatic brain injury with cognitive disability: a voxel-wise analysis of diffusion tensor imaging. J Neurotrauma. 2008;25(11):1335-1342.

14. Cantu RC. Concussion classification: ongoing controversy. In: Slobounov S, Sebastianelli W, editors. Foundations of Sports-Related Brain Injuries. New York, NY: Springer Science and Business Media, Inc.; 2006:87-110.

15. Bazarian JJ, Zhong J, Blyth B, Zhu T, Kavcic V, Peterson D. Diffusion tensor imaging detects clinically important axonal damage after mild traumatic brain injury: a pilot study. J Neurotrauma. 2007;24(9): 1447-1459.

16. Basser PJ, Pierpaoli C. Microstructural and physiological features of tissues elucidated by quantitative-diffusion-tensor MRI. J Magn Reson B. 1996;111(3):209-219.

17. Naeser MA, Saltmarche A, Krengel MH, Hamblin MR, Knight JA. Improved cognitive function after transcranial, light-emitting diode treatments in chronic, traumatic brain injury: two case reports. Photomed Laser Surg. 2011;29(5):351-358.

18. Greicius MD, Krasnow B, Reiss AL, Menon V. Functional connectivity in the resting brain: a network analysis of the default mode hypothesis. Proc Natl Acad Sci U SA. 2003;100(1):253-258.

19. Greicius $M$. Resting-state functional connectivity in neuropsychiatric disorders. Curr Opin Neurol. 2008;21(4):424-430.

20. Singh KD, Fawcett IP. Transient and linearly graded deactivation of the human default-mode network by a visual detection task. Neuroimage. 2008;41(1):100-112.

21. Bonnelle V, Leech R, Kinnunen KM, et al. Default mode network connectivity predicts sustained attention deficits after traumatic brain injury. J Neurosci. 2011;31(38):13442-13451. 
22. Sherman LE, Rudie JD, Pfeifer JH, Masten CL, McNealy K, Dapretto M. Development of the default mode and central executive networks across early adolescence: a longitudinal study. Dev Cogn Neurosci. 2014;10: $148-159$

23. Bonnelle V, Ham TE, Leech R, et al. Salience network integrity predicts default mode network function after traumatic brain injury. Proc Natl Acad Sci U S A. 2012;109(2):4690-4695.

24. Menon V, Uddin LQ. Saliency, switching, attention and control: a network model of insula function. Brain Struct Funct. 2010;214(5-6):655-667.

25. Wang X, Dmochowski J, Husain M, et al. Transcranial infrared brain stimulation modulates EEG alpha power. Brain Stimulat. 2017;10(4):e67-e69.

26. Vargas E, Barrett DW, Saucedo CL, et al. Beneficial neurocognitive effects of transcranial laser in older adults. Lasers Med Sci 2017;32(5):1153-1162.

27. Hamblin MR. Shining light on the head: photobiomodulation for brain disorders. BBA Clin. 2016;6:113-124.

28. De Freitas LF, Hamblin MR. Proposed mechanisms of photobiomodulation or low-level light therapy. IEEE J Sel Top Quantum Electron 2016;22(3):7000417.

29. Wan S, Parrish JA, Anderson RR, Madden M. Transmittance of nonionizing radiation in human tissues. Photochem Photobiol. 1981;34:679-681.

30. Pitzschke A, Lovisa B, Seydoux O, et al. Red and NIR light dosimetry in the human deep brain. Phys Med Biol. 2015;60(7):2921-2937.

31. Whelan HT, Smits RL Jr, Buchman EV, et al. Effects of NASA lightemitting diode irradiation on wound healing. J Clin Laser Med Surg. 2001;19:305-314.

32. Oron U, Yaakobi T, Oron A, et al. Attenuation of infarct size in rats and dogs after myocardial infarction by low-energy laser irradiation. Lasers Surg Med. 2001;28(3):204-211.

33. Naeser M, Ho M, Martin PE, et al. Improved language after scalp application of red/near-infrared light-emitting diodes: pilot study supporting a new, noninvasive treatment for chronic aphasia. Proc Soc Behav Sci. 2012;61:138-139.

34. Xuan W, Vatansever F, Huang L, Hamblin MR. Transcranial low-level laser therapy enhances learning, memory, and neuroprogenitor cells after traumatic brain injury in mice. J Biomed Opt. 2014;19(10):108003.

35. Desmet KD, Paz DA, Corry JJ, et al. Clinical and experimental applications of NIR-LED photobiomodulation. Photomed Laser Surg. 2006;24(2):121-128.

36. Wang X, Tian F, Soni SS, Gonzalez-Lima F, Liu H. Interplay between up-regulation of cytochrome-c-oxidase and hemoglobin oxygenation induced by near-infrared laser. Sci Rep. 2016;6:30540.

37. Wang X, Tian F, Reddy DD, et al. Up-regulation of cerebral cytochromec-oxidase and hemodynamics by transcranial infrared laser stimulation: a broadband near infrared spectroscopy study. J Cereb Blood Flow Metab. 2017;37(12):3789-3802.

38. Tian F, Hase SN, Gonzalez-Lima F, Liu H. Transcranial laser stimulation improves human cerebral oxygenation. Lasers Surg Med. 2016;48(4):343-349.

39. Khuman J, Zhang J, Park J, Carroll JD, Donahue C, Whalen MJ. Lowlevel laser light therapy improves cognitive deficits and inhibits microglial activation after controlled cortical impact in mice. J Neurotrauma 2012;29(2):408-417.

40. Sompol P, Xu Y, Ittarat W, Daosukho C, St Clair D. NF-kappa B-associated MnSOD induction protects against beta-amyloid-induced neuronal apoptosis. J Mol Neurosci. 2006;29:279-288.

41. Wang X, Reddy DD, Nalawade SS, Pal S, Gonzalez-Lima F, Liu H. Impact of heat on metabolic and hemodynamic changes in transcranial infrared laser stimulation measured by broadband near-infrared spectroscopy. Neurophotonics. 2017;5(1):011004.

42. Richardson RM, Sun D, Bullock MR. Neurogenesis after traumatic brain injury. Neurosurg Clin NAm. 2007;18(1):169-181.

43. Naeser MA, Martins PI, Ho MD, et al. Transcranial, red/near-infrared light-emitting diode therapy to improve cognition in chronic traumatic brain injury. Photomed Laser Surg. 2016;34(12):610-626.
44. Xuan W, Agrawal T, Huang L, Gupta GK, Hamblin MR. Low-level laser therapy for traumatic brain injury in mice increases brain derived neurotrophic factor (BDNF) and synaptogenesis. J Biophotonics. 2015;8(6):502-511.

45. Blanco NJ, Saucedo CL, Gonzalez-Lima F. Transcranial infrared laser stimulation improves rule-based, but not information-integration, category learning in humans. Neurobiol Learn Mem. 2017;139: 69-75.

46. Cui X, Bray S, Bryant DM, Glover GH, Reiss AL. A quantitative comparison of NIRS and fMRI across multiple cognitive tasks. Neuroimage. 2011;54(4):2808-2821.

47. Haeussinger FB, Heinzel S, Hahn T, Schecklmann M, Ehlis AC, Fallgatter AJ. Simulation of near-infrared light absorption considering individual head and prefrontal cortex anatomy: implications for optical neuroimaging. PLoS One. 2011;6(10):e26377.

48. Strangman GE, Zhang Q, Li Z. Scalp and skull influence on near infrared photon propagation in the Colin 27 brain template. Neuroimage. 2014;85(Pt 1):136-149.

49. Okada E, Delpy DT. Near-infrared light propagation in an adult head model. II. Effect of superficial tissue thickness on the sensitivity of the near-infrared spectroscopy signal. Appl Opt. 2003;42(16): $2915-2922$.

50. Jagdeo JR, Adams LE, Brody NI, Siegel DM. Transcranial red and near infrared light transmission in a cadaveric model. PLoS One. 2012;7(10):e47460.

51. Tedford CE, DeLapp S, Jacques S, Anders J. Quantitative analysis of transcranial and intraparenchymal light penetration in human cadaver brain tissue. Lasers Surg Med. 2015;47(4):312-322.

52. Hashmi JT, Huang Y-Y, Sharma SK, et al. Effect of pulsing in low-level light therapy. Lasers Surg Med. 2010;42(6):450-466.

53. Barrett DW, Gonzalez-Lima F. Transcranial infrared laser stimulation produces beneficial cognitive and emotional effects in humans. Neuroscience. 2013;230:13-23.

54. Blanco NJ, Maddox WT, Gonzalez-Lima F. Improving executive function using transcranial infrared laser stimulation. J Neuropsychol. 2017;11(1):14-25.

55. Oron A, Oron U, Streeter J, et al. Low-level laser therapy applied transcranially to mice following traumatic brain injury significantly reduces long-term neurological deficits. $J$ Neurotrauma. 2007;24(4): 651-656.

56. Wu Q, Huang Y-Y, Dhital S, et al. Low level laser therapy for traumatic brain injury. SPIE Proc. 2010;7552:755206-755208.

57. Naeser MA, Zafonte R, Krengel MH, et al. Significant improvements in cognitive performance post-transcranial, red/near-infrared light-emitting diode treatments in chronic, mild traumatic brain injury: open-protocol study. J Neurotrauma. 2014;31(11):1008-1017.

58. Bogdanova Y, Martin P, Ho MD, et al. LED therapy improves sleep and cognition in chronic moderate TBI: pilot case studies. Arch Phys Med Rehabil. 2014;95:e77.

59. Henderson TA, Morries LD. SPECT perfusion imaging demonstrates improvement of traumatic brain injury with transcranial near-infrared laser phototherapy. Adv Mind Body Med. 2015;29(4):27-33.

60. Lapchak PA. Transcranial near-infrared laser therapy applied to promote clinical recovery in acute and chronic neurodegenerative diseases. Expert Rev Med Devices. 2012;9(1):71-83.

61. Zivin JA, Albers GW, Bornstein N, et al. Effectiveness and safety of transcranial laser therapy for acute ischemic stroke. Stroke 2009;40(4):1359-1364.

62. Hashmi JT, Huang Y-Y, Osmani BZ, Sharma SK, Naeser MA, Hamblin MR. Role of low-level laser therapy in neurorehabilitation. $P M R$. 2010;2(12 Suppl 2):S292-S305.

63. U.S. Food and Drug Administration [webpage on Internet]. Silver Spring: Guidance for Industry and FDA Staff - Class II Special Controls Guidance Document: Low Level Laser System for Aesthetic Use [cited April 14, 2011]. Available from: https://www.fda.gov/RegulatoryInformation/Guidances/ucm251260.htm\#5. Accessed November 10, 2017. 
64. dos Santos JGRP, Paiva WS, Teixeira MJ. The current role of noninvasive treatments in traumatic brain injury. J Neurol Disord. 2016; 4:294.

65. Morries LD, Cassano P, Henderson TA. Treatments for traumatic brain injury with emphasis on transcranial near-infrared laser phototherapy. Neuropsychiatr Dis Treat. 2015;11(1):2159-2175.
66. Neville IS, Hayashi CY, Hajj SAE, et al. Repetitive transcranial magnetic stimulation (rTMS) for the cognitive rehabilitation of traumatic brain injury (TBI) victims: study protocol for a randomized controlled trial. Trials. 2015;16:440.

67. Demirtas-Tatlidede A, Vahabzadeh-Hagh AM, Pascual-Leone A. Can noninvasive brain stimulation enhance cognition in neuropsychiatric disorders? Neuropharmacology. 2013;64:566-578.

\section{Publish your work in this journal}

Medical Devices: Evidence and Research is an international, peerreviewed, open access journal that focuses on the evidence, technology, research, and expert opinion supporting the use and application of medical devices in the diagnosis, monitoring, treatment and management of clinical conditions and physiological processes. The identification of novel devices and optimal use of existing devices which will lead to improved clinical outcomes and more effective patient management and safety is a key feature. The manuscript management system is completely online and includes a quick and fair peer-review system. Visit http://www. dovepress.com/testimonials.php to read real quotes from authors.

Submit your manuscript here: https://www.dovepress.com/medical-devices-evidence-and-research-journal 